\title{
A Shared Service Algorithm for a Trunked Mobile System
}

\author{
YURDAER N. DOGANATA, STUDENT MEMBER, IEEE
}

\begin{abstract}
A model has been developed and solved which captures the essence of the concept of mobile dispatch service. There are two types of users, dispatch and interconnect (calls routed from the public switched telephone network, of mach longer holding times). Also, only some of the available repeaters can be used for interconnect traffic, and blocked interconnect calls are cleared. Dispatch cals, however, can access all repeaters, and then are queued if all repeaters are busy. One design decision involves a trade between blocking of interconnect calls and queueing of dispatch calls. A formula has been derived for both interconnect blocking probability and the mean dispatch queueing time to permit this trade. The results indicate that a good operating point is to have $\left(N-a_{d}\right)$ (rounded down) interconnected repeaters, where there are $N$ total repeaters and $a_{d}$ Erlangs of dispatch traffic.
\end{abstract}

\section{INTRODUCTION}

$I^{N}$ $N$ THE LAST SEVERAL years, centralized interconnect trunked systems have been introduced to service both dispatch-type mobile users and telephone line users [1]. The term "trunked" means that the telephone line is interfaced to the radio system at the repeater, which allows both dispatch and telephone line users to access the system simultaneously. Thus, two different types of communications utilize the same system facilities. As far as communications traffic is concerned, the basic difference between these two types of users is their average holding (service) times. A typical dispatch communication is conducted between the members of a mobile fleet or between the base station and mobiles. These are very short messages compared to an average telephone conversation, such as conducted by interconnect users. We call the telephone line users interconnect and the dispatch type mobile users dispatch users.

The purpose of this study is to increase the performance of a mobile trunked system for a given traffic demand, in other words to decrease both average dispatch delay and the blocking probability of interconnect traffic.

Fig. 1 shows the basic components of the trunked mobile system. We note that only a fraction of the repeaters can be physically connected to interconnect calls. Therefore, the number of repeaters available for interconnect users is restricted. On the other hand, dispatch users have the ability to access the system from any one of the repeaters.

In any trunking system which serves both interconnect and dispatch users, it is thus important for the system to be able to intelligently allocate the valuable air time resources between the two. "Intelligently" means the decision about the channel

Manuscript received October 1, 1985; revised May 3, 1986.

The author is with the Department of Electrical Engineering, California Institute of Technology, Pasadena, CA 91125.

IEEE Log Number 8610657.

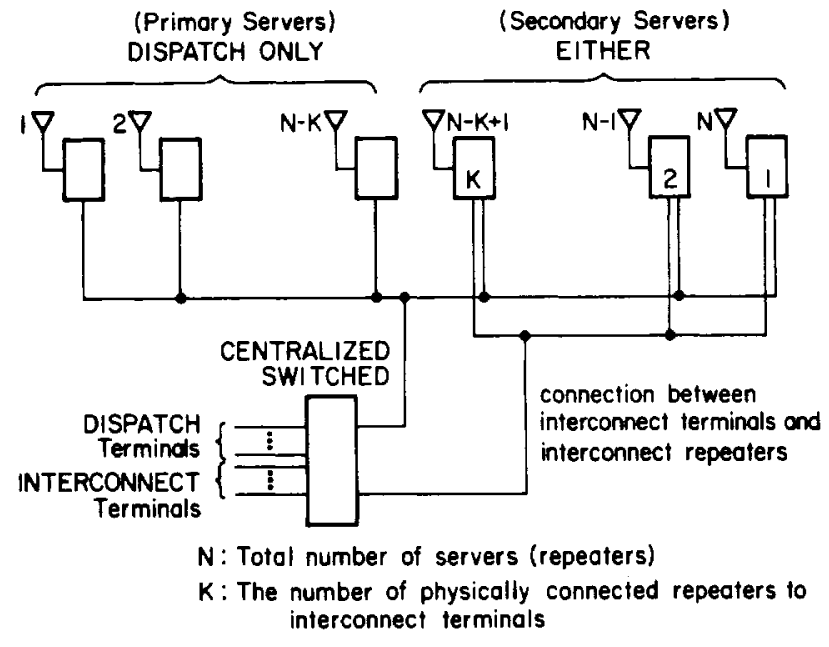

Fig. 1. Basic components of a trunked mobile system.

resources to be allocated is based on the current traffic demands. The parameters related to the current traffic are measured periodically and channel resources are reallocated in order to optimize the system performance until the next measurement.

As an example, in the evening when dispatch traffic is usually light and several channels are likely to be available at any given point in time, it makes sense for interconnect users to be able to utilize these available channels. On the other hand, in the morning, when dispatch traffic is at a peak and the dispatch demand is greater relative to the interconnect demand than usual, it makes sense that channels previously available for interconnect users be switched temporarily to dispatch use only until the peak subsides.

In this study, a service sharing algorithm is proposed and analysed based on a comparison of the blocking probability for interconnect users and the average dispatch delay. The next section describes the traffic model of this system.

\section{System Traffic Model}

In developing a sharing algorithm for a mobile trunked system, the following model is used.

i) The arrivals of dispatch and interconnect call requests are independent and are assumed to be coming from memoryless (Poisson) sources [3] of infinite population.

ii) In both traffic streams, the call durations are exponentially distributed. That is, service times are memoryless and independent of everything else. (The average call duration for dispatch calls will later be assumed less than that for interconnect calls.) 
iii) We call the interconnected repeaters secondary servers and the remaining (dispatch-only) repeaters primary servers. When a call request from an interconnect user arrives, it is lost (blocked calls cleared) if the secondary servers are busy, i.e., cleared from the system. If the primary servers are busy but there are empty secondary servers, the dispatch arrival is served, otherwise it is placed in an infinite queue. Fig. 2 shows a flow diagram of this trunked mobile traffic.

iv) The mean service time for interconnect traffic $1 / \mu_{i}$ is much longer than the mean service time for dispatch traffic, $1 /$ $\mu_{d}$, i.e.,

$$
1 / \mu_{i} \gg 1 / \mu_{d} .
$$

v) Incoming dispatch call requests search for an empty repeater starting from the leftmost repeater, whereas incoming interconnect calls start from rightmost repeater, i.e., if there is more than one empty repeater, dispatch calls use the leftmost, and interconnect calls use the rightmost. (The reason for this allocation is to avoid dispatch calls occupying secondary servers when a primary would do. Otherwise, the blocking probability of interconnect calls would increase beyond that intended.)

\section{ANALysis of TRAFFiC}

Using the fact that average holding time of an interconnect call is much larger than the dispatch holding time, the conditional steady state probabilities of dispatch users can be found simply by employing the results of basic queueing theory. But in order to find the mean dispatch delay we need to find the steady state probabilities of interconnect users as well. It will be seen that the steady state probabilities of interconnect users depend on the conditional blocking probabilities of interconnect users. Thus, if we know these conditional blocking probabilities, we can find the steady state interconnect probabilities, which then yield the mean dispatch delay and blocking probability of an interconnect request. Possible blocking conditions of interconnect calls and how to calculate them are given in Section IV. The details of the analysis are given below.

We have an $N$-server queueing system, where $N$ is the total number of repeaters. We will view the number of servers (repeaters) occupied by dispatch users as a random process that varies according to how many servers are assigned to interconnect traffic. We define the "interconnect state" as the number of interconnect-occupied servers. The interconnect state varies much more slowly than the dispatch state, which is the total number of dispatch-occupied servers. This is simply because

$$
\frac{1}{\mu_{i}} \gg \frac{1}{\mu_{d}} .
$$

It is therefore a good approximation to assume that the time spent in each interconnect state is much longer than the time it takes the number of dispatch requests present in the system to reach steady-state behavior given the number of interconnect calls in progress. This "steady-state" approximation of Honig [2] is the basis for our analysis. Given that $i$ servers are currently assigned to interconnect traffic, the probability that

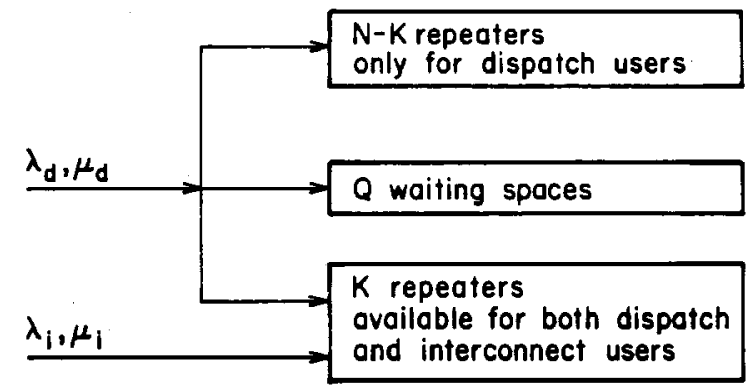

$\lambda_{d}$ : arrival rate of dispatch calls

$\lambda_{i}$ : arrival rate of interconnect calls

$\mu_{d}:$ service rate of dispatch calls

$\mu_{i}$ : service rate of interconnect colls

Fig. 2. Flow diagram of trunked mobile traffic.

there are $k$ dispatch calls in the system is simply found by analyzing the $M / M / N-i$ queueing system [4].

We define the conditional state probabilities of dispatch traffic as

\section{$P_{d / i}(k)=\operatorname{Pr}$ (there are $k$ dispatch users in the system, given that $i$ servers are occupied by interconnect calls).}

From the assumed equilibrium at steady state, we have

$$
\begin{gathered}
\lambda_{d} P_{d / i}(0)=\mu_{d} P_{d / i}(1) \\
\lambda_{d} P_{d / i}(1)=2 \mu_{d} P_{d / i}(2) \\
\vdots \\
\lambda_{d} P_{d / i}(N-i-1)=(N-i) \mu_{d} P_{d / i}(N-i) .
\end{gathered}
$$

These equations yield

$$
P_{d / i}(k)=P_{d / i}(0) a_{d}^{k} \frac{1}{k !} ; \quad 0 \leq k \leq N-i
$$

For $k>N-i$, however, the arriving dispatch requests are queued, so the steady-state probabilities satisfy

$$
\begin{gathered}
\lambda_{d} P_{d / i}(N-i)=(N-i) \mu_{d} P_{d / i}(N-i+1) \\
\lambda_{d} P_{d / i}(N-i+1)=(N-i) \mu_{d} P_{d / i}(N-i+2) .
\end{gathered}
$$

These equations imply that

$$
P_{d / i}(k)=P_{d / i}(0) a_{d}^{k} \frac{(N-i)^{N-i-k}}{(N-i) !} ; \quad k \geq N-i,
$$

where

$$
P_{d / i}(0)=\left[\sum_{k=0}^{N-i-1} \frac{a_{d}^{k}}{k !}+\frac{1}{(N-i) !} a_{d}^{N-i} \frac{1}{1-\frac{a_{d}}{(N-i)}}\right]^{-1}
$$

and

$$
a_{d}=\frac{\lambda_{d}}{\mu_{d}}
$$

is the dispatch traffic. 
Given that there are $i$ assigned interconnect calls, the mean number of queued dispatch requests is readily found by using (2) and (3):

$$
\begin{gathered}
E(q / i)=\sum_{q=1}^{\infty} q P_{d / i}(N-i+q) \\
=\frac{P_{d / i}(0)}{(N-i) !} a_{d}^{N-i} \sum_{q=1}^{\infty} q \frac{a_{d}^{q}}{(N-i)^{q}} \\
E(q / i)=\frac{P_{d / i}(0)}{(N-i) !} a_{d}^{N-i} \frac{\frac{a_{d}}{(N-i)}}{\left[1-\frac{a_{d}}{(N-i)}\right]^{2}} .
\end{gathered}
$$

From this, the mean number of queued dispatch requests is found as follows:

$$
E(q)=\sum_{l=0}^{K} P_{I}(l) E(q / l)
$$

where $K$ is the number of interconnect repeaters and $P_{I}(l)$ is the probability of having $l$ interconnect users in the system. This probability can be found by using the method described in [2], which we now describe.

$P_{\Gamma}(l)$ depends not only on the previous interconnect state but also on the number of dispatch calls occupying the secondary servers. Assuming there are $l$ interconnect users in the system, where $l<K$, an arriving interconnect request is blocked if the remaining $K-l$ secondary servers are all occupied by dispatch calls. Therefore, the transition rate to the $(l+1)$ th state is the arrival rate times the entrance probability, which we define as

$$
\begin{aligned}
& \phi_{l}=\operatorname{Pr} \text { (a new interconnect request is able to be } \\
& \text { served, given that there are } l \text { interconnect } \\
& \text { calls in the system), for } 0 \leq l \leq K .\left(\phi_{\mathbf{K}}\right. \text { is, } \\
& \text { of course, } 0 .)
\end{aligned}
$$

The rest of the analysis is similar to the analysis of an $M / M / K$ blocking system except that the arrival rates in each state $l$ are decreased by the factor $\phi_{l}$.

Assuming exponential service times and using the equilibrium equations, $P_{I}(l)$ can be found as follows:

$$
\begin{gathered}
\lambda_{i} P_{I}(0) \phi_{0}=\mu_{i} P_{I}(1) \\
\lambda_{i} P_{I}(1) \phi_{1}=2 \mu_{i} P_{I}(2) \\
\vdots \\
\lambda_{i} P_{I}(K-1) \phi_{(K-1)}=K \mu_{i} P_{I}(K) .
\end{gathered}
$$

Therefore,

$$
\begin{aligned}
P_{I}(l) & =\frac{P_{I}(0)}{l !} a_{i}^{l} \phi_{0} \phi_{1} \cdots \phi_{l-1} \\
& =\frac{P_{I}(0)}{l !} a_{i}^{l} \sum_{k=0}^{l-1} \phi_{k},
\end{aligned}
$$

where

$$
a_{i}=\frac{\lambda_{i}}{\mu_{i}}
$$

the offered interconnect traffic, and

$$
P_{l}(0)=\left[\sum_{m=0}^{K} \frac{a_{i}^{m}}{m !} \prod_{j=0}^{m-1} \phi_{j}\right]^{-1}
$$

Finally, substituting (7) into (6),

$$
P_{I}(l)=\frac{\frac{a_{i}^{l}}{l !} \prod_{j=0}^{l-1} \phi_{j}}{\sum_{m=0}^{K-1} \frac{a_{i}^{m}}{m !} \prod_{j=0}^{m-1} \phi_{j}} .
$$

The blocking probability of interconnect users can be found by employing the following equation:

$$
P_{B}=\sum_{l=0}^{K-1} P_{B / l} P_{I}(l)+P_{I}(K)
$$

where

$$
\begin{aligned}
& P_{B / l}=\operatorname{Pr} \text { (an arriving interconnect request is blocked, } \\
& \text { given that there are } l \text { interconnect calls in } \\
& \text { the system). }
\end{aligned}
$$

Here

$$
P_{B / l}=1-\phi_{l}
$$

Therefore, (9) can be written by employing (10) as

$$
P_{B}=\sum_{l=0}^{K-1}\left(1-\phi_{l}\right) P_{I}(l)+P_{I}(K) .
$$

As is seen from (5), (8) and (11), the blocking probability for interconnect users and the expected number of dispatch users waiting for service depend on the entrance probabilities $\phi_{l}$ or on the conditional probabilities $P_{B / l}$. The next section describes how to calculate these conditional blocking probabilities, thus solving our problem.

\section{Blocking Conditions for Interconnect Traffic}

It is clear that an incoming interconnect call is blocked precisely when all the secondary servers are busy. The secondary servers are busy if

(i) There are already $K$ interconnect calls in the system, where $K$ is the total number of repeaters connectable to interconnect calls, or

(ii) The number of dispatch users $d$ in the system is greater than or equal to $N-i$, when there are $i$ interconnect users in the system and $0 \leq i \leq K-1$, or

(iii) The number of dispatch users $d$ in the primary (dispatch-only) repeaters is less than $\mathbf{N}-\mathbf{K}$, but there is at least one dispatch user in the secondary repeaters and the rest of the secondary repeaters are occupied by interconnect calls. 


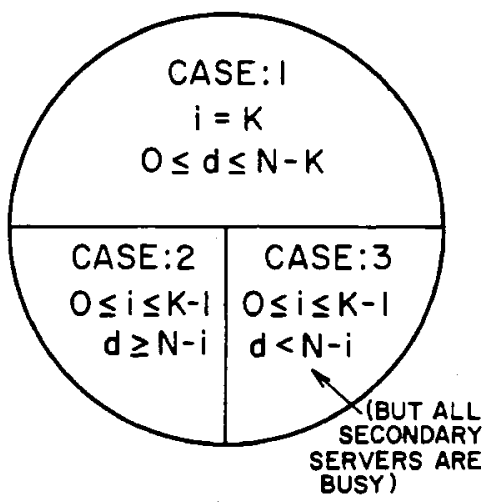

Fig. 3. Possible blocking cases for interconnect users when all the secondary repeaters are busy.

Possible blocking cases for interconnect users are illustrated in Fig. 3. Obviously, these are disjoint events. One possible evolution of these blocking conditions is illustrated below. In the following, $\mathbf{D}$ corresponds to a dispatch user, I corresponds to an interconnect user and - corresponds to empty server. Here time increases as the index $j$ on $t_{j}$ increases; $N=15, K$ $=5$.

(i) $i=K$

$i$ : the number of interconnect users in the system

$$
\begin{aligned}
& t_{1} \rightarrow \mathbf{D} \cdot \mathbf{D} \text { D D D D D D D D D I I I I I }
\end{aligned}
$$

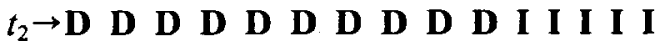

$$
\begin{aligned}
& t_{3} \rightarrow \mathbf{D} \text { D }
\end{aligned}
$$

(ii) $d \geq N-i$

$d$ : number of dispatch users in the system; $i=3$

$$
\begin{aligned}
& t_{1} \rightarrow \mathbf{D} \text { D D D D D D D D D D D D D I I D D I }
\end{aligned}
$$

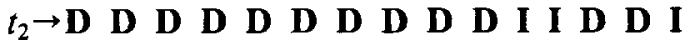

(iii) $d+i=K$ in the secondary repeaters, while $d<N-$ $K$ in the primary; $i=\mathbf{3}$

$$
\begin{aligned}
& t_{1} \rightarrow \mathbf{D} \\
& t_{2} \rightarrow \mathbf{D}
\end{aligned}
$$

If all the repeaters were physically connected to the interconnect terminals, i.e. $N=K$, then the only blocking condition would be $d \geq N-i$ as described in (ii). This is the case where all arrivals can access any repeater. Such a case has been analysed by Honig [2]. But for a trunked mobile system the first and the third conditions must also be considered.

Define the probabilities corresponding to the events described in (i), (ii), and (iii) as follows:

$P_{1}=\operatorname{Pr}$ (there are $K$ interconnect calls in the system)

$P_{2 / i}=\operatorname{Pr}$ (the number of dispatch users $d$ is greater than or equal to $N-i$ given that there are $i$ interconnect calls in the system)

$P_{2 / i}=P_{d / i}(d \geq N-i)$

$P_{3 / i}=\operatorname{Pr}$ (The number of dispatch users in the primary is less than $N-K$, given that there are $i$ interconnect calls and $K-i$ dispatch calls in the secondary, where $i>0$ ).

$P_{2 / i}$ is obtained easily by using (2):

$$
\begin{aligned}
P_{2 / i} & =\sum_{j=N-i}^{\infty} P_{d / i}(j) \\
& =P_{d / i}(0) \frac{(N-i)^{N-i}}{(N-i) !} \frac{1}{1-\frac{a_{d}}{(N-i)}} .
\end{aligned}
$$

(Note that $a_{d} /(N-i)<1$, otherwise an infinite queue forms in steady state.) Finally, $P_{1}$ is found from

$$
P_{1}=P_{I}(K)
$$

by definition, the probability that there are $K$ interconnect calls in the system.

The conditional blocking probability is written by using the previous definitions as

$$
P_{B / i}=P_{2 / i}+P_{3 / i}, \quad 0 \leq i<K
$$

so the entrance probability $\phi_{i}$ is

$$
\phi_{i}=1-P_{2 / i}-P_{3 / i}, \quad 0 \leq i<K .
$$

Now the remaining problem is to calculate $P_{3 / i}$. The next section is devoted to this calculation.

\section{Calculation of $P_{3 / i}$}

In the beginning, it was assumed that, if there is more than one empty repeater, an arriving dispatch call request uses the leftmost repeater. Therefore, if the event described in (iii) occurs, then in one of the past repeater states the number of dispatch users must have been equal to $N-i$. The following diagram shows the state of repeaters at times $t_{1}, t_{2}, \cdots, t_{6}$ and illustrates the blocking condition described by (iii).

At times $t_{2}, t_{3}, t_{4}, t_{5}$ and $t_{6}$, the interconnect requests are blocked, even though there are empty primary servers. The system is in a blocking state between $t_{2}$ and $t_{6}$ as is described in (iii). But before entering this state, the system comes from another blocking state as described in (ii). At time $t_{1}$, the system is in such a state and $d=N-i$. When the system is in blocking condition (iii), there are $i$ interconnect and $K-i$ dispatch users in the secondary servers and they therefore occupy all secondary servers. On the other hand, the number of dispatch users in the primary servers may vary from 0 to $N$ - $K-1$. Whenever a departure occurs in the secondary servers, the system gets out of the blocking state.

Since all secondary servers are busy and there is no dispatch user in the queue for this particular case (iii), the number of dispatch users in the primary servers describes the system completely. So, we define the state of the repeaters as the 


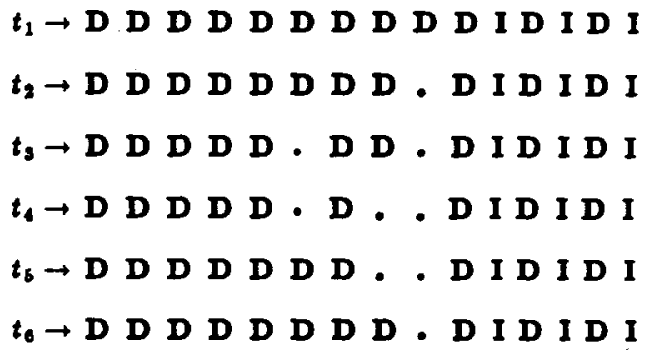

Fig. 4. State of repeaters in blocking condition (iii).

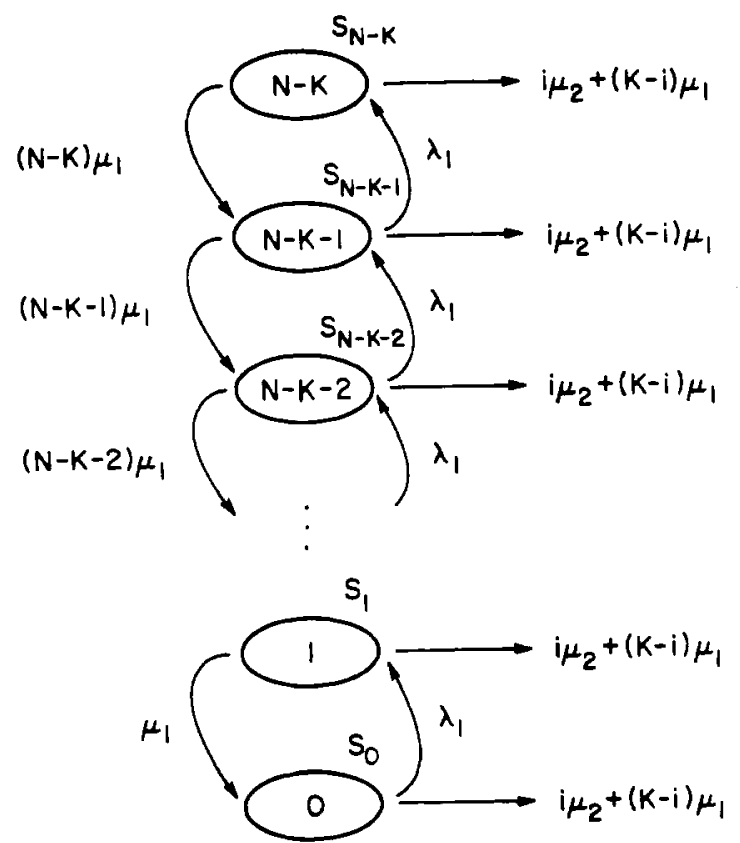

Fig. 5. State transition diagram when secondary servers are busy.

number of dispatch users in the primary servers. We let $X(t)$ denote this state at time $t$. The random process $X(t)$ forms a continuous-time Markov chain, because the future value of $X(t)$ depends on the past only through its current value. Fig. 5 is the state-transition-rate diagram for this process. Concentrating on state $S_{j}$, we observe that it can be entered only from state $S_{(j-1)}$ or state $S_{(j+1)}$, and similarly, it can be left only by entering state $S_{(j-1)}$ or state $S_{(j+1)}$, or by getting out of the blocking conditions. At steady state, the rate at which the system leaves $S_{j}$ must be equal to the rate at which the system goes into that state, because $X(t)$ is an ergodic Markov chain and therefore its steady-state probabilities exist.

Now define the conditional state probabilities as follows, for $0 \leq i \leq K$ :

$Q_{j / i}=\operatorname{Pr}$ (There are $j$ dispatch users in the primary, given that all secondary servers are busy and there are $i$ interconnect users in the secondary).

Then for $j=N-K$,

$$
\begin{aligned}
Q_{j / i} & =Q_{(N-K) / i} \\
& =P_{d / i}(N-i) \\
& =P_{d / i}(0) \frac{a_{d}^{(N-i)}}{(N-i) !} .
\end{aligned}
$$

The blocking condition described in (iii) occurs when the system is in one of the states described above and $j<N-K$. Therefore

$$
P_{3 / i}=\sum_{m=0}^{N-K-1} Q_{m / i}, \quad 0 \leq i<K
$$

Specifically, if we focus on state $S_{j}$ we observe that the rate at which probability "flows" into this state at time $t$ is given by flow rate into $S_{j}=(j+1) \mu_{d} Q_{(j+1) / i}+\lambda_{d} Q_{(j-1) / i}$ whereas the flow rate out of that state at time $t$ is given by flow rate out of $S_{j}=\left(j \mu_{d}+A_{i}\right) Q_{j / i}$ where $A_{i}=\lambda_{d}+i \mu_{i}+$ $(K-i) \mu_{d}$.

Clearly, these two rates must be equal to each other, that is,

$$
\begin{gathered}
\mu_{d} Q_{1 / i}=A_{i} Q_{0 / 1} \\
2 \mu_{d} Q_{2 / i}+\lambda_{d} Q_{0 / i}=\left(\mu_{d}+A_{i}\right) Q_{1 / i} \\
\vdots \\
(N-K) \mu_{d} Q_{(N-K) / i}+\lambda_{d} Q_{(N-K-2) / i} \\
=\left((N-K-1) \mu_{d}+A_{i}\right) Q_{(N-K-1) / i} .
\end{gathered}
$$

Note that $Q_{(N-K) / i}=P_{d / i}(N-i)$.

In matrix form these relationships can be written as

$$
\begin{gathered}
\left(\begin{array}{ccccc}
\delta_{0} & -\mu_{d} & 0 & \cdots & 0 \\
-\lambda_{d} & \delta_{1} & -2 \mu_{d} & \cdots & 0 \\
0 & -\lambda_{d} & \delta_{2} & \cdots & 0 \\
\vdots & \vdots & \vdots & \ddots & \vdots \\
0 & 0 & 0 & \cdots & \delta_{M}
\end{array}\right) \quad\left(\begin{array}{c}
Q_{0 / i} \\
Q_{1 / i} \\
Q_{2 / i} \\
\vdots \\
Q_{M / i}
\end{array}\right) \\
\quad=\left(\begin{array}{c}
0 \\
0 \\
\vdots \\
(M+1) \mu_{d} Q_{(M+1) / i}
\end{array}\right)
\end{gathered}
$$

where $M=N-K-1$ and $\delta_{j}=j \mu_{d}+A_{i}$.

This system of linear equations is easily solved by using the Gauss elimination method. The result is

$$
Q_{r / i} . C_{r}=(r+1) \mu_{d} Q_{(r+1) / i}, \quad 0 \leq r \leq N-K-1
$$

where $C_{r}$ is given as

$$
C_{r}=\left(\delta_{r}-\frac{r \mu_{d} \lambda_{d}}{C_{r-1}}\right), \quad C_{0}=\delta_{0} .
$$

Thus,

$$
\begin{gathered}
Q_{(N-K-1) / i}=\frac{(N-K) \mu_{d}}{C_{N-K-1}} P_{d / i}(N-i) \\
Q_{(N-K-2) / i}=\frac{(N-K)(N-K-1) \mu_{d}^{2}}{C_{N-K-1} C_{N-K-2}} P_{d / i}(N-i) \\
\vdots \\
Q_{0 / i}=\frac{(N-K) ! \mu_{d}^{(N-K)}}{C_{N-K-1} C_{N-K-2} \cdots C_{0}} P_{d / i}(N-i)
\end{gathered}
$$


where

$$
P_{d / i}(N-i)=P_{d / i}(0) \frac{a_{d}^{N-i}}{(N-i)} .
$$

$P_{3 / i}$ can now be determined by employing (20).

$$
P_{3 / i}=\sum_{m=0}^{N-K-1} Q_{m / i} \quad 0 \leq i<K .
$$

\section{OUTLINE OF THE RESULTS}

Our aim is to find the blocking probability of interconnect users and the average dispatch delay as a function of the number of interconnected repeaters. A closed-form expression does not seem useful (or possible) here. Instead $P_{3 / i}, P_{I}(l), \phi_{i}$ are computed and the blocking probability of interconnect users is obtained by making proper substitutions.

The outline of this computation algorithm will now be derived. The conditional blocking probability of interconnect users is given as

$$
P_{B / i}=\left\{\begin{array}{ll}
P_{2 / i}+P_{3 / i}, & 0 \leq i<K \\
P_{I}(K), & i=K
\end{array}\right\} .
$$

$P_{3 / i}$ is computed as described above and $\phi_{i}$ is simply found by the definition

$$
\phi_{i}=1-P_{2 / i}-P_{3 / i} .
$$

$P_{2 / i}$ is given as in (12)

$$
P_{2 / i}=P_{d / i}(0) \frac{(N-i)^{N-i}}{(N-i) !} \frac{1}{1-\frac{a_{d}}{(N-i)}} .
$$

$P_{I}(K)$ is found using the method described in Section III as

$$
P_{I}(K)=\frac{\frac{a_{i}^{K}}{K !} \prod_{j=0}^{K-1} \phi_{j}}{\sum_{m=0}^{K-1} \frac{a_{i}^{m}}{m !} \prod_{j=0}^{K-1} \phi_{j}}
$$

After computing the steady state probability $P_{I}(i)$ and conditional blocking probability $\boldsymbol{P}_{B / i}$ of interconnect users, the blocking probability $P_{B}$ is obtained by employing (9):

$$
P_{B}=\sum_{l=0}^{K-1} P_{B / l} P_{I}(l)+P_{I}(K)
$$

where $P_{I}(l)$ is

$$
P_{I}(l)=\frac{\frac{a_{i}^{l}}{l !} \prod_{j=0}^{l-1} \phi_{j}}{\sum_{m=0}^{K-1} \frac{a_{i}^{m}}{m !} \prod_{j=0}^{K-1} \phi_{j}} .
$$

The mean number of queued dispatch requests is found as

$$
E(q)=\sum_{l=0}^{K} P_{I}(l) E(q / l)
$$

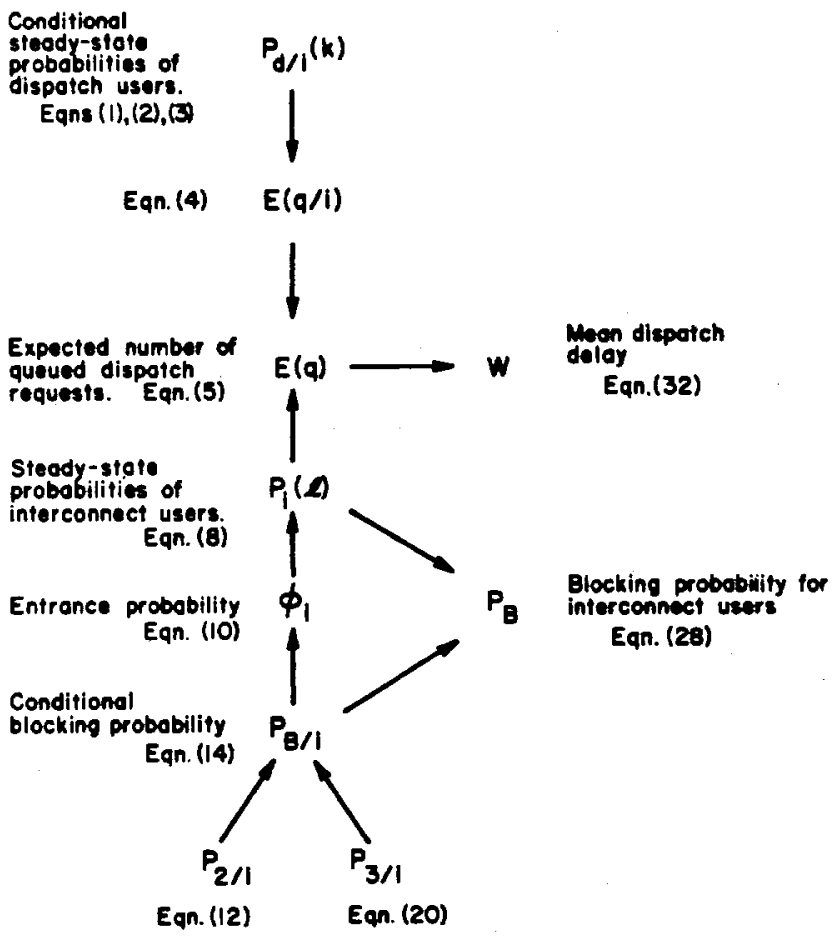

Fig. 6. Hierarchy in computation.

where $E(q / l)$ is

$$
E(q / l)=P_{d / i}(0) \frac{a_{d}^{N-l}}{(N-l) !} \frac{\frac{a_{d}}{(N-l)}}{\left[1-\frac{a_{d}}{(N-l)}\right]^{2}}
$$

and $P_{I}(l)$ is as given in (29). The average dispatch delay is then obtained by employing Little's formula [4]:

$$
W=E(q) / \lambda_{d}
$$

where $W$ is the mean delay and $E(q)$ is the mean number of dispatch users in the queue given in (30). Fig. 6 describes the hierarchy of our computations.

Fig. 7 compares the interconnect blocking probability with the average delay for various traffic levels as computed from (28) and (32). The number of repeaters connectable to interconnect calls $K$ is shown in the abscissa. The ordinate corresponds to blocking probability or mean dispatch delay, respectively. Obviously, when $K=0$, the blocking probability of interconnect users is 1 and the mean dispatch delay is at its minimum. In this case, all repeaters are reserved for dispatch traffic. As we increase $K$, interconnect users start to access the repeaters that were previously reserved for dispatch traffic and the blocking probability of interconnect users decreases. Increasing the dispatch traffic increases the mean dispatch delay, which shifts the delay curve left and the blocking probability curve up. When the amount of dispatch traffic approaches the number of repeaters reserved for dispatch users, the mean delay increases rapidly. The service rates used are $\mu_{d}=4.01$ and $\mu_{i}=0.2$, which correspond to the mean dispatch service time of $14.96 \mathrm{~s}$ and interconnect service time of $5 \mathrm{~min}$. The arrival rates for the first 


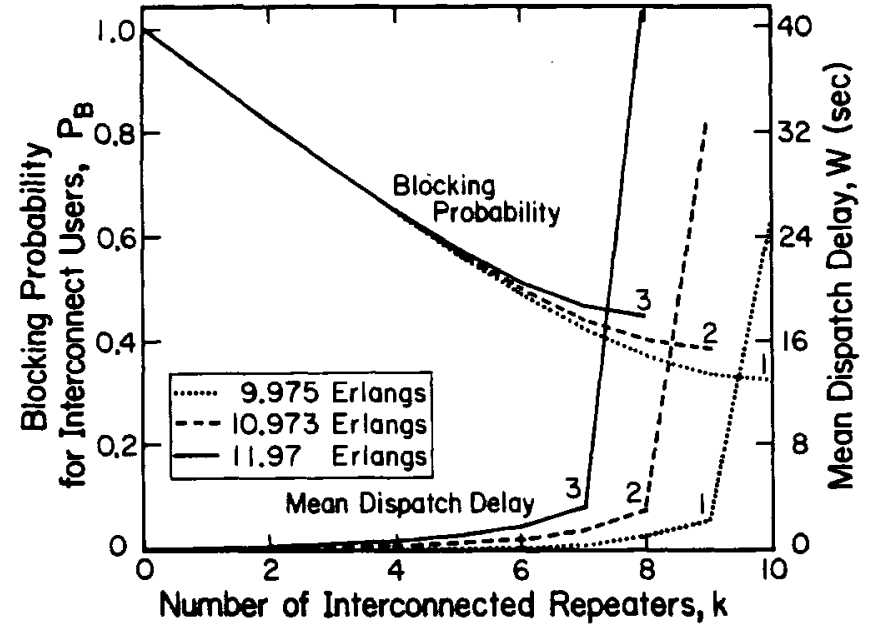

Fig. 7. The interconnect blocking probability and mean dispatch delay as a function of the number of interconnected repeaters.

comparison are $\lambda_{d}=40$ customers per minute and $\lambda_{i}=2$ customers per minute, for the second comparison $\lambda_{d}=44$ and for the last one $\lambda_{d}=48$.

\section{Conclusion}

At the beginning of this study we proposed that a sharing algorithm for a trunked mobile system could be developed which would trade off the interconnect blocking probability against the average dispatch delay. The blocking probability for interconnect users and mean dispatch delay are found as described in the previous section. As a result of the comparison of these quantities, it is seen that the interconnect blocking probability decreases almost linearly as $K$, the number of secondary servers, increases and it is independent of dispatch traffic for small values of $K$. When $K$ is small and the dispatch traffic is light, secondary repeaters are not likely to be occupied by dispatch users, so the probability of an interconnect user being served is not affected by dispatch traffic and is nearly proportional to the number of secondary servers. As $K$ increases, the dispatch users start to use the secondary servers, so the probability of being blocked depends not only on the number of servers but also on the number of dispatch calls in the secondary servers. Thus, the decrease in blocking probability for interconnect users is not linear anymore.

Since the average holding time of an interconnect call is much larger than the average holding time of a dispatch call, the interconnect state varies very slowly. As a result of this, when the number of interconnect users in the system is equal to $K$ and the dispatch traffic is greater than $N-K$, the number of dispatch users waiting for service starts to increase and queue peaks occur as expected. As must be, this causes an increase in the average delay of dispatch calls. However, the mean delay is almost negligible when the number of primary servers is greater than the amount of dispatch traffic, because the probability of finding an empty repeater is very high for an incoming dispatch call. For example, when the dispatch traffic is 9.975 Erlangs and $K=10$, the mean delay is $26 \mathrm{~s}$ as is seen from the second curve. But if $K$ is decreased by one, that is, if the number of primary servers is increased by one, the mean delay suddenly drops to $2.4 \mathrm{~s}$; for $K=8$ it is $1.35 \mathrm{~s}$.

As a result, for small values of $K$, as $K$ increases the blocking probability decreases considerably and the mean delay remains negligible. But as soon as $N-K$ approaches the value of the dispatch traffic, the mean delay becomes very large whereas the blocking probability for interconnect calls does not vary much.

So in order to decrease the blocking probability of interconnect users and to keep the mean dispatch delay below a certain level, we conclude that the number of secondary repeaters should be the largest possible value smaller than $\left(N-a_{d}\right)$ where $N$ is the total number of repeaters and $a_{d}$ is the dispatch traffic. As an example, let $a_{d}=9.975$ Erlangs, $N=20, N-$ $a_{d}=10.025$. According to our criteria the value of $K$ must be at most ten, otherwise the dispatch queue blows up. In other words, a good operating point is to have $N-a_{d}$ (rounded down) interconnect repeaters. This could have been approximately predicted in advance, as our above heuristics show.

\section{ACKNOWLEDGMENT}

The author would like to express his gratitude to Professor E. C. Posner for his appreciable supervision and encouragement throughout this work. Special thanks are also extended to Dr. Garry Hess and Dr. Jim Mikulski from MOTOROLA Communications and Electronics, Inc., Schaumburg, for providing the necessary documents about the subject.

\section{REFERENCES}

[1] Motorola Product Digest, "Centralized interconnect for $800 \mathrm{MHz}$ trunked systems," Motorola Commun. Electron., Schaumburg, IL, 1978.

[2] M. L. Honig, "Analysis of TDMA network with voice and data traffic," AT\&T Bell Lab. Tech. J., vol. 63, no. 8, Oct. 1984.

[3] G. Hess and J. Cohn, "Communication load and delay in mobile trunked systems," in Proc. 3Ist IEEE Veh. Technol. Conf., Washington, DC, Apr. 1981, p. 271.

[4] L. Kleinrock, Queueing Systems, Volume I: Theory. New York: Wiley, 1975.

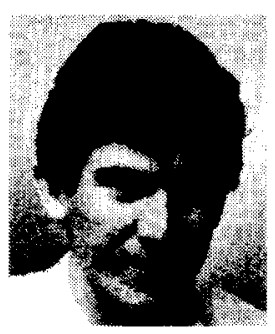

Yurdaer N. Doganata (S'83) was born in Turkey, on June 27,1959 . He received the B.S. and M.S. degrees in electrical engineering from Middle East Technical University, Ankara, Turkey, in 1981 and 1983, respectively.

Currently, he is pursuing the Ph.D. degree in electrical engineering at the Calfornia Institute of Technology, Pasadena. His research involves traffic management for new services such as trunked mobile radio. 